\title{
A Ring-Focus Antenna with Splash Plate in Ka-Band
}

\author{
Laiding Zhao $\mathbb{D}^{1,2}$ Xiaodong Bai, ${ }^{1,2}$ Rui Li, ${ }^{1,3}$ and Jidong Xie ${ }^{1,2}$ \\ ${ }^{1}$ College of Telecommunications and Information Engineering, Nanjing University of Posts and Telecommunications, \\ Nanjing 210003, China \\ ${ }^{2}$ Satellite Communication and Navigation Collaborative Innovation Center, Nanjing 210003, China \\ ${ }^{3}$ Electronic and Optical Engineering \& College of Microelectronics and Information Engineering, Nanjing University of Posts and \\ Telecommunications, Nanjing 210003, China
}

Correspondence should be addressed to Laiding Zhao; zhaold@njupt.edu.cn

Received 5 September 2017; Revised 27 November 2017; Accepted 21 December 2017; Published 18 March 2018

Academic Editor: Xuejun Lu

Copyright (C) 2018 Laiding Zhao et al. This is an open access article distributed under the Creative Commons Attribution License, which permits unrestricted use, distribution, and reproduction in any medium, provided the original work is properly cited.

\begin{abstract}
A design for Ka-band satellite communication antenna in earth station is introduced. This kind of antenna uses splash plate feed based on ring-focus antenna. The theory of the new splash plate feed design is presented and the relative equations are derived. The measured antenna parameters, VSWR, and patterns are highly consistent with the simulated results, which demonstrates the success of the design for such kind of antenna that can be effectively used in Ka-band applications.
\end{abstract}

\section{Introduction}

There are many kinds of Ka-band antenna, as reported in documents, including microstrip array antenna [1-3], waveguide array antenna [4-6], waveguide slot antenna [7], horn antenna [8-10], dielectric spherical lens feed antenna [11-13], and reflector antenna $[14,15]$. The efficiency of Kaband antenna is usually in the range of 50\% to $60 \%$ [16].

The antenna designers always pursue high gain while using low sidelobes for the design of Ka-band antenna [17-27].

Ring-focus parabolic antenna overcomes feed shelter to realize low sidelobes and VSWR in a broad bandwidth and has been applied widely in VSAT. The antenna can enhance its efficiency by changing the shape of hat feed [28], reducing its size by using a spline-profiled smooth horn [29], and improving its performance on VSWR and crosspolarization via a small ring-shaped aperture [30]. But all the schemes above have low efficiency due to the support for subreflector.

Splash plate feed, which traces back to World War II, has not been used widely for the high VSWR [31]. Since the 1980s, antenna with splash plate feed has been researched by few papers, which present the power distribution control and equiphase on the main reflector aperture through dielectric materials and subreflector forming, while the main attention is payed on subreflector forming to improve performance [32-36]. Since the antenna with splash plate feed does not need the support for subreflector, it can gain higher efficiency. However, the problem caused by feed shelter [32-36] still exists.

In this paper, the design of a new antenna in Ka-band is introduced. This antenna adopts splash plate feed, whose main reflector is ring focus without strut and feed shelter.

In this paper, the principle of ring-focus reflector antenna with splash plate feed is introduced firstly. A group of differential equations are derived and the geometry of the antenna's subreflector and dielectric surface is obtained by solving these differential equations. The simulated results indicate that this antenna can achieve high gain with low sidelobes. This kind of antenna has been produced based on the proposed design in this paper. The measured farfield patterns are highly consistent with the simulated results.

This product can be applied widely in Ka-band satellite communications considering its novel structure, low cost, good electrical performance, and high efficiency. 


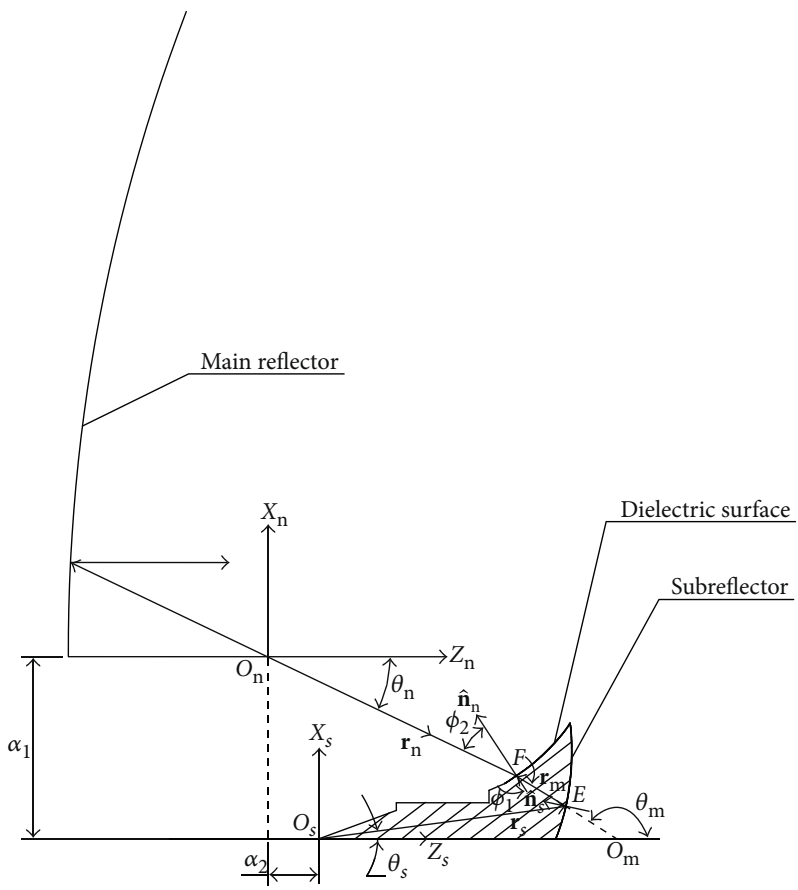

Figure 1: Principle of ring-focus antenna with splash plate feed.

\section{Theoretical Analysis}

The method based on geometric optics is adopted to design the ring-focus antenna with splash plate feed. This antenna consists of four parts including the main reflector, splash plate feed, media, and subreflector.

This antenna is rotational symmetric, and the principle of such design is simply described through $X Z 2 \mathrm{D}$ section in Figure 1.

The main reflector of such antenna is ring focus. It is produced by rotation of a parabola, whose focal length is $F$, away from symmetry axis $a_{1}$. The main reflector equation of this antenna, whose focus is the origin, can be expressed as follows:

$$
Z=\frac{\left(|X|-a_{1}\right)^{2}}{4 F}-F .
$$

The principle of designed antenna is shown in Figure 1. The feed phase center is $O_{s}$. The optics ray is from $O_{s}$ to $E$, then reflects to $F$, and then refracts to $O_{n}$, where $O_{n}$ is the ring focal of the main reflector. $O_{m}$ is a point of intersection by reflect line extension line and $Z_{s}$ axis. The vector $\mathbf{r}_{s}$ is pointing from $O_{s}$ to $E$. The vector $\mathbf{r}_{m}$ is pointing from $E$ to $F$. The vector $\mathbf{r}_{n}$ is pointing from $O_{n}$ to $F$.

2.1. Design of Subreflector. 3D description equations are required in theory derivation while $2 \mathrm{D}$ section is shown Figure 1. Thus, assuming that $\phi_{s}$ is the included angle of vector $\mathbf{r}_{s}$ in $O_{s} X_{s} Z_{s}$ plane and $O_{s} X_{s}$ axis, if $f_{s}\left(\theta_{s}, \phi_{s}\right)$ is the equation of subreflector, the vector $\mathbf{r}_{s}$ can be expressed as

$$
\mathbf{r}_{s}=f_{s}\left(\theta_{s}, \phi_{s}\right) \widehat{\mathbf{r}}_{s}
$$

where $\widehat{\mathbf{r}}_{s}$ is the unit vector. In rectangular coordinate system, $\widehat{\mathbf{r}}_{s}$ and $\widehat{\mathbf{r}}_{m}$ can be expressed as

$$
\begin{gathered}
\widehat{\mathbf{r}}_{s}=\sin \theta_{s}\left(\cos \phi_{s} \widehat{\mathbf{x}}_{s}+\sin \phi_{s} \widehat{\mathbf{y}}_{s}\right)+\cos \theta_{s} \widehat{\mathbf{z}}_{s} \\
\widehat{\mathbf{r}}_{m}=\sin \theta_{m}\left(\cos \phi_{s} \widehat{\mathbf{x}}_{s}+\sin \phi_{s} \widehat{\mathbf{y}}_{s}\right)+\cos \theta_{m} \widehat{\mathbf{z}}_{s} .
\end{gathered}
$$

To express simply, let $f_{s}\left(\theta_{s}, \phi_{s}\right)=f_{s}$. Then the differential coefficient is

$$
\begin{aligned}
\frac{d \widehat{\mathbf{r}}_{s}}{d \phi_{s}}= & \left(\frac{d f_{s}}{d \phi_{s}} \sin \theta_{s} \cos \phi_{s}-f_{s} \sin \theta_{s} \sin \phi_{s}\right) \widehat{\mathbf{x}}_{s} \\
& +\left(\frac{d f_{s}}{d \phi_{s}} \sin \theta_{s} \sin \phi_{s}+f_{s} \sin \theta_{s} \cos \phi_{s}\right) \widehat{\mathbf{y}}_{s} \\
& +\frac{d f_{s}}{d \phi_{s}} \cos \theta_{s} \widehat{\mathbf{z}}_{s}, \\
\frac{d \widehat{\mathbf{r}}_{s}}{d \theta_{s}}= & \left(\frac{d f_{s}}{d \theta_{s}} \sin \theta_{s} \cos \phi_{s}+f_{s} \cos \theta_{s} \cos \phi_{s}\right) \widehat{\mathbf{x}}_{s} \\
& +\left(\frac{d f_{s}}{d \theta_{s}} \sin \theta_{s} \sin \phi_{s}+f_{s} \cos \theta_{s} \sin \phi_{s}\right) \widehat{\mathbf{y}}_{s} \\
& +\left(\frac{d f_{s}}{d \theta_{s}} \cos \theta_{s}-f_{s} \sin \theta_{s}\right) \widehat{\mathbf{z}}_{s} .
\end{aligned}
$$

The unit normal vector of subreflector is

$$
\widehat{\mathbf{n}}_{s}=\frac{\left(d \mathbf{r}_{s} / d \phi_{s}\right) \times\left(d \mathbf{r}_{s} / d \theta_{s}\right)}{\left|\left(d \mathbf{r}_{s} / d \phi_{s}\right) \times\left(d \mathbf{r}_{s} / d \theta_{s}\right)\right|},
$$

where " $x$ " represents vector cross product. It can be derived from (5) that 


$$
\begin{aligned}
\widehat{\mathbf{n}}_{s}= & \frac{1}{\Delta}\left(\frac{d f_{s}}{d \theta_{s}} \cos \theta_{s}-f_{s} \sin \theta_{s}\right) \cos \phi_{s} \widehat{\mathbf{x}}_{s} \\
& +\left(\frac{d f_{s}}{d \theta_{s}} \cos \theta_{s}-f_{s} \sin \theta_{s}\right) \sin \phi_{s} \widehat{\mathbf{y}}_{s} \\
& -\left(\frac{d f_{s}}{d \theta_{s}} \sin \theta_{s}+f_{s} \cos \theta_{s}\right) \widehat{\mathbf{z}}_{s},
\end{aligned}
$$

where

$$
\Delta=\sqrt{\left(\frac{d f_{s}}{d \theta_{s}}\right)^{2}+f_{s}^{2}}
$$

The inner product of incident wave $\mathbf{r}_{s}$ 's unit vector and subreflector's unit normal vector is

$$
-\widehat{\mathbf{r}}_{s} \cdot \widehat{\mathbf{n}}_{s}=\frac{f_{s}}{\sqrt{\left(d f_{s} / d \theta_{s}\right)^{2}+f_{s}^{2}}}
$$

The inner product of reflected wave $\mathbf{r}_{m}$ from subreflector and subreflector's unit normal vector is

$$
\widehat{\mathbf{r}}_{m} \cdot \widehat{\mathbf{n}}_{s}=\frac{\left(d f_{s} / d \theta_{s}\right) \sin \left(\theta_{m}-\theta_{s}\right)-f_{s} \cos \left(\theta_{m}-\theta_{s}\right)}{\sqrt{\left(d f_{s} / d \theta_{s}\right)^{2}+f_{s}^{2}}} .
$$

According to Snell's law,

$$
-\widehat{\mathbf{r}}_{s} \cdot \widehat{\mathbf{n}}_{s}=\widehat{\mathbf{r}}_{m} \cdot \widehat{\mathbf{n}}_{s} .
$$

It can be derived from (8), (9), and (10) that

$$
\frac{d \mathbf{r}_{s}}{d \theta_{s}}=\mathbf{r}_{s}\left(\theta_{s}\right) \cot \left(\frac{\theta_{m}-\theta_{s}}{2}\right)
$$

2.2. Design of Dielectric Surface Forming. For simple derivation, assuming a coordinate system $O_{n} X_{n} Z_{n}$, the dielectric surface equation is

$$
\mathbf{r}_{n}=f_{n}\left(-\theta_{n}, \phi_{s}\right) \widehat{\mathbf{r}}_{n}
$$

where $\widehat{\mathbf{r}}_{n}$ is the unit vector and can be expressed as

$$
\widehat{\mathbf{r}}_{n}=-\sin \theta_{n}\left(\cos \phi_{s} \widehat{\mathbf{x}}_{n}+\sin \phi_{s} \widehat{\mathbf{y}}_{n}\right)+\cos \theta_{n} \widehat{\mathbf{z}}_{n}
$$

in a rectangular coordinate system. For simple description, let $f_{n}\left(-\theta_{n}, \phi_{s}\right)$ be $f_{\mathrm{n}}$; the unit normal vector of the dielectric surface is

$$
\widehat{\mathbf{n}}_{n}=\frac{\left(d \mathbf{r}_{n} / d \phi_{n}\right) \times\left(d \mathbf{r}_{n} / d \theta_{n}\right)}{\left|\left(d \mathbf{r}_{n} / d \phi_{n}\right) \times\left(d \mathbf{r}_{n} / d \theta_{n}\right)\right|}
$$

So it can be derived that

$$
\begin{aligned}
\widehat{\mathbf{n}}_{n}= & \frac{1}{\Delta^{\prime}}\left(\frac{d f_{n}}{d \theta_{n}} \cos \theta_{n}+f_{n} \sin \theta_{n}\right) \cos \phi_{s} \widehat{\mathbf{x}}_{n} \\
& +\left(\frac{d f_{n}}{d \theta_{n}} \cos \theta_{n}-f_{n} \sin \theta_{n}\right) \sin \phi_{s} \widehat{\mathbf{y}}_{n} \\
& +\left(\frac{d f_{n}}{d \theta_{n}} \sin \theta_{n}-f_{n} \cos \theta_{n}\right) \widehat{\mathbf{z}}_{n},
\end{aligned}
$$

where

$$
\Delta^{\prime}=\sqrt{\left(\frac{d f_{n}}{d \theta_{n}}\right)^{2}+f_{n}^{2}}
$$

The inner product of incident wave $\mathbf{r}_{m}$ 's unit vector and dielectric surface's unit normal vector is

$$
\widehat{\mathbf{r}}_{m} \cdot \widehat{\mathbf{n}}_{n}=\frac{\left(d f_{n} / d \theta_{n}\right) \sin \left(\theta_{m}+\theta_{n}\right)-f_{n} \cos \left(\theta_{m}+\theta_{n}\right)}{\sqrt{\left(d f_{n} / d \theta_{n}\right)^{2}+f_{n}^{2}}}=\cos \phi_{1} .
$$

The inner product of refracted wave $\mathbf{r}_{n}$ and dielectric surface's unit normal vector is

$$
\left(-\widehat{\mathbf{r}}_{n}\right) \cdot \widehat{\mathbf{n}}_{n}=\frac{f_{n}}{\sqrt{\left(d f_{n} / d \theta_{n}\right)^{2}+f_{n}^{2}}}=\cos \phi_{2} .
$$

According to Snell's law,

$$
\frac{\sin \phi_{1}}{\sin \phi_{2}}=\frac{1}{n}
$$

where

$$
n=\sqrt{\varepsilon_{\mathrm{r}}} .
$$

It is derived from (17), (18), and (19) that

$$
\frac{d f_{n}}{d \theta_{n}}=\frac{n f_{n} \sin \left(\theta_{m}+\theta_{n}\right)}{1-n \cos \left(\theta_{m}+\theta_{n}\right)}
$$

2.3. Energy Conservation Equation. According to the law of energy conservation,

$$
P\left(\theta_{s}\right) \sin \theta_{s} d \theta_{s}=\frac{-I\left(X_{m}\right) X_{m} d X_{m}}{A},
$$

where $P\left(\theta_{s}\right)$ is the spatial angle distribution function of feed axisymmetric radiation power, $I\left(X_{m}\right)$ is the aperture power distribution function of the antenna main paraboloid, $X_{m}$ is the radius variable of the antenna aperture, and $A$ is the proportionality coefficient.

The main reflector equation of ring-focus reflector antenna is

$$
X_{m}=2 F \tan \frac{\theta_{n}}{2}+a_{1}
$$


In logs, it can be obtained as

$$
\frac{d X_{m}}{d \theta_{n}}=F \sec ^{2} \frac{\theta_{n}}{2}
$$

According to (23) and (24), then

$$
\frac{d \theta_{n}}{d \theta_{s}}=-\frac{A P\left(\theta_{s}\right) \sin \theta_{s} \cos ^{2}\left(\theta_{n} / 2\right)}{F I\left(X_{m}\right) X_{m}} .
$$

2.4. Equiphase Equation. In definition, $\theta_{s \max }$ is the maximum of $\theta_{s}, f_{s \max }$ is the maximum of $f_{s}, \theta_{n \min }$ is the minimum of $\theta_{s}$, and $f_{n \max }$ is the maximum of $f_{n}$. According to the qualification requirement, $P\left(\theta_{s}\right), I\left(X_{m}\right), a_{1}, a_{2}, \theta_{s \max }$, $f_{s \max }$, and $F$ can be certain firstly.

When $\theta_{s}=\theta_{s \max }$ and $f_{s}=f_{s \max }$, boundary condition can be obtained according to the following geometrical relationship:

$$
\begin{aligned}
\theta_{n \min } & =\arctan \frac{f_{s \max } \cos \theta_{s \max }+a_{2}}{a_{1}-f_{s \max } \sin \theta_{s \max }}, \\
f_{n \max } & =\sqrt{\left(f_{s \max } \cos \theta_{s \max }+a_{2}\right)^{2}+\left(f_{s \max } \sin \theta_{s \max }-a_{1}\right)^{2}} .
\end{aligned}
$$

In a dielectric material, the optical length that an electromagnetic wave radiates from primary feed $O_{s}$ and refract to the air after being reflected by subreflector to the main reflector's focus is equal. Namely,

$$
\begin{aligned}
& n f_{s}+n f_{m}+f_{n}=K, \\
& f_{s \text { max }}+f_{n \text { max }}=K .
\end{aligned}
$$

2.5. Calculation of Subreflector Equation and Dielectric Surface 2D Equation. According to the geometric relationship in Figure 1, it can be obtained that

$$
\begin{aligned}
& f_{s} \sin \theta_{s}+f_{m} \sin \left(\pi-\theta_{m}\right)+f_{n} \sin \theta_{n}=a_{1}, \\
& f_{s} \cos \theta_{s}+a_{2}=f_{m} \cos \left(\pi-\theta_{m}\right)+f_{n} \cos \theta_{n} .
\end{aligned}
$$

When $\theta_{s}$ is certain, $\theta_{m}, \theta_{n}, f_{s}, f_{m}$, and $f_{n}$ can be solved according to (11), (21), (23), (25), and (28).

In $O_{s} X_{s} Z_{s}$ plane, subreflector equations are

$$
\begin{aligned}
& X=f_{s} \sin \theta_{s}, \\
& Z=f_{s} \cos \theta_{s} .
\end{aligned}
$$

Dielectric surface equations are

$$
\begin{aligned}
& X=\alpha_{1}-f_{n} \sin \theta_{n}, \\
& Z=f_{n} \cos \theta_{n}-\alpha_{2} .
\end{aligned}
$$

Thus, the shapes of subsurface and dielectric surface can be obtained.

\section{Structure of Antenna}

The main reflector, whose material is carbon fiber, is a rotationally symmetric paraboloid. The surface of the reflector should be processed by metallization, and the accuracy

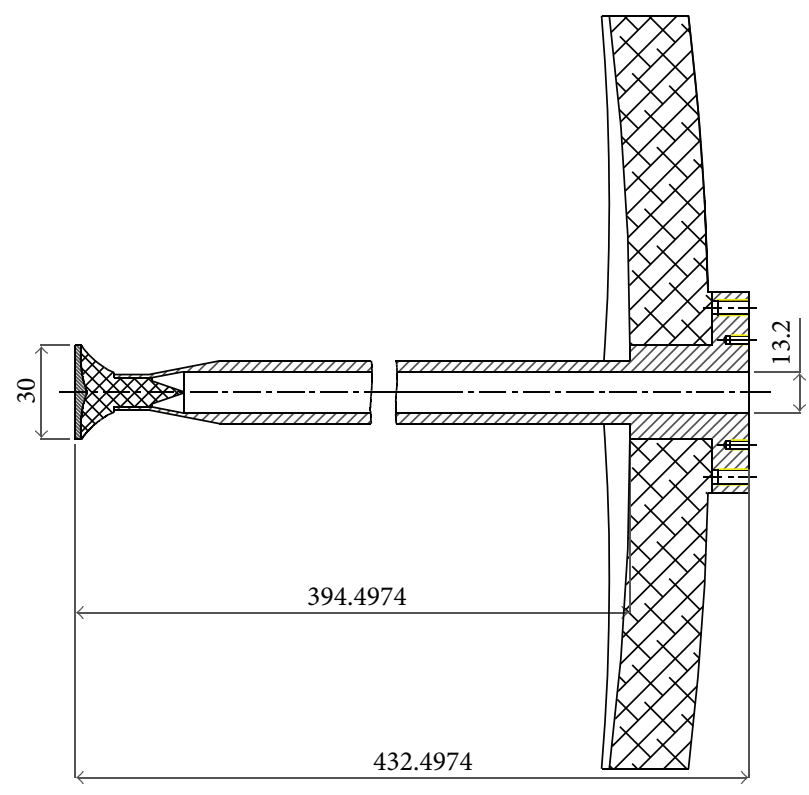

FIGURE 2: Structure of the main and side reflector.

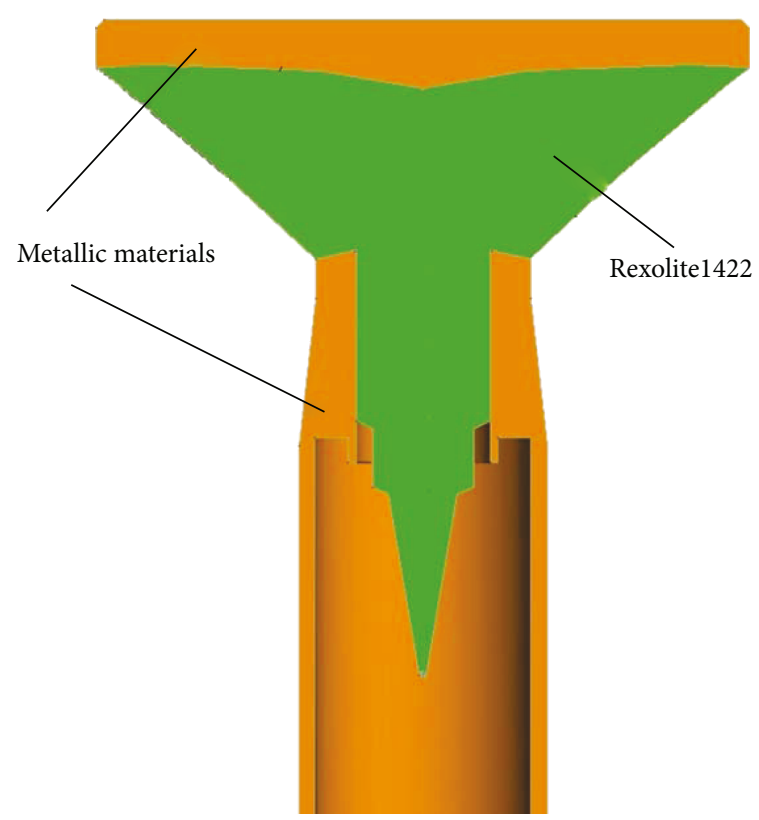

Figure 3: Exploded view of the feed structure.

should be controlled within $0.3 \mathrm{~mm}$ (r.m.s.). In the processing, a carbon fiber reflector and a copper sheet are made out of molds. Then they are bonded together with glue. The accuracy of the molds ensures the accuracy of the reflector. The structures of the main reflector and subreflector and dielectric are shown in Figure 2.

The exploded view of the feed structure is shown in Figure 3, where the green and yellow parts are dielectric material REXOLITE1422 and aluminum material, respectively. After programming according to the designed data, the subreflector and dielectric can be processed by high 


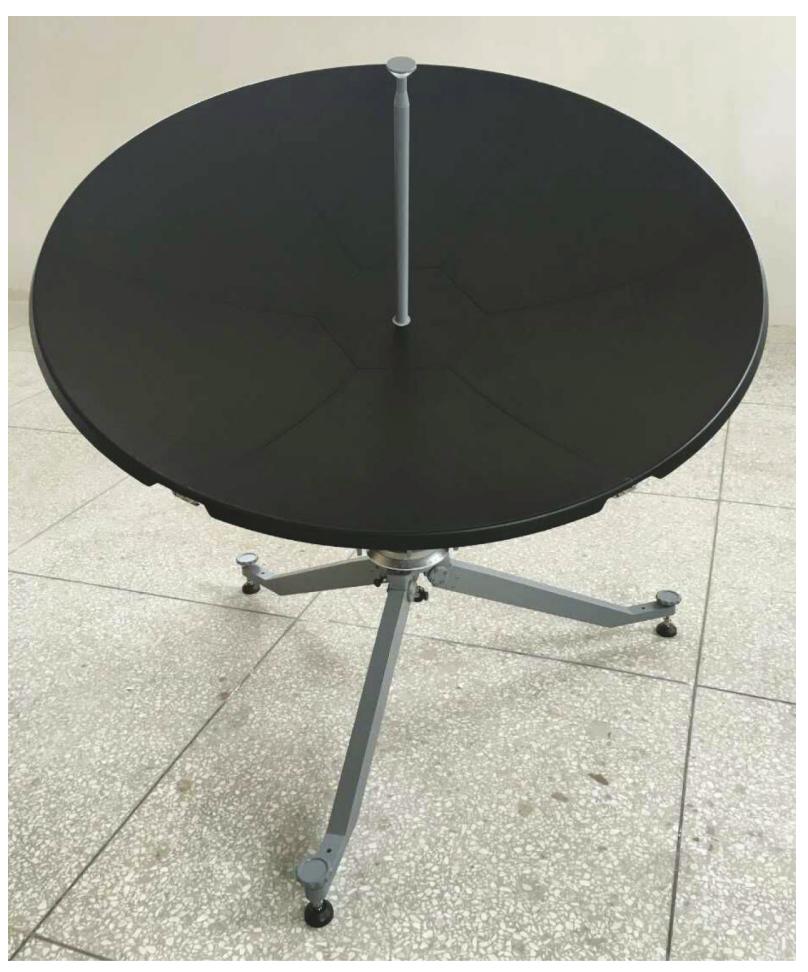

FIgURE 4: Antenna front view.

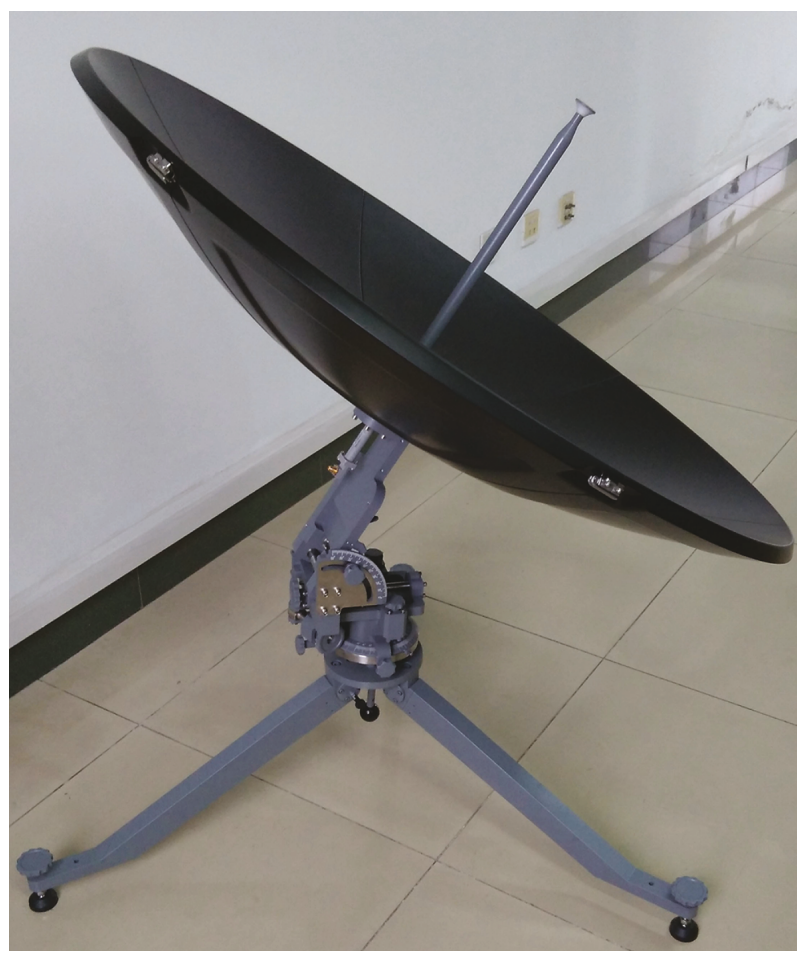

FIgURE 5: Antenna lateral view.

precision CNC (computer numerical control) machines. Ultraviolet (UV) glue is adopted here to bond the subreflector and the dielectric. Since the dielectric is transparent, the glue between subreflector and dielectric will cure after UV radiates for a period of time.

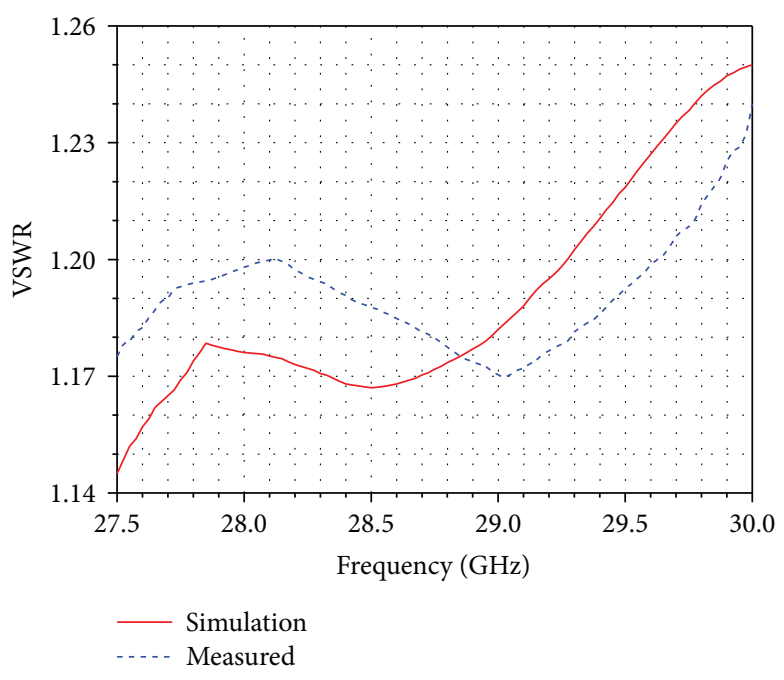

FIGURE 6: Curves of transmitting VSWR.

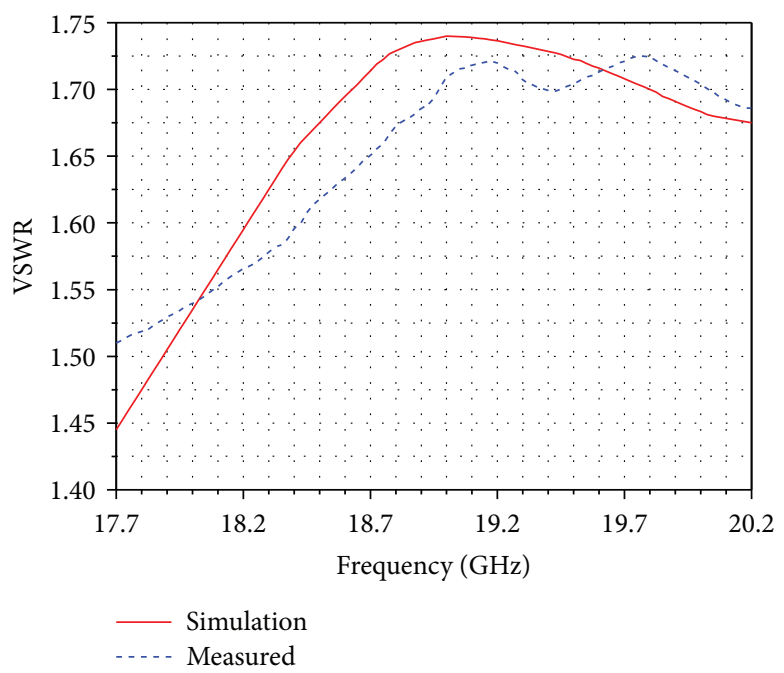

Figure 7: Curve of receiving VSWR.

Let $15 \mathrm{~mm} \leq|X| \leq 525 \mathrm{~mm}, \quad F=420 \mathrm{~mm}, \quad a_{1}=15 \mathrm{~mm}$. After fabrication of the subreflector and dielectric materials, the assembled antenna is shown in Figures 4 and 5.

\section{Simulation and Measurement on VSWR}

This antenna adopts circular polarization. It uses 27.5 30.0 GHz as transmitting frequency and $17.7 \sim 20.2 \mathrm{GHz}$ as receiving frequency. Physical optics (PO) method and finite element method (FEM) are combined in the simulation by FEKO, where PO method is used for the main reflector and FEM for the feed (subreflector and dielectric). The simulation defines "mesh" as $1 / 6$ wavelength. The computer has an 8-core CPU and 128 GB RAM.

The curves of VSWR are obtained through the simulation of FEKO, and the simulated results are shown as red continuous lines in Figures 6 and 7. 


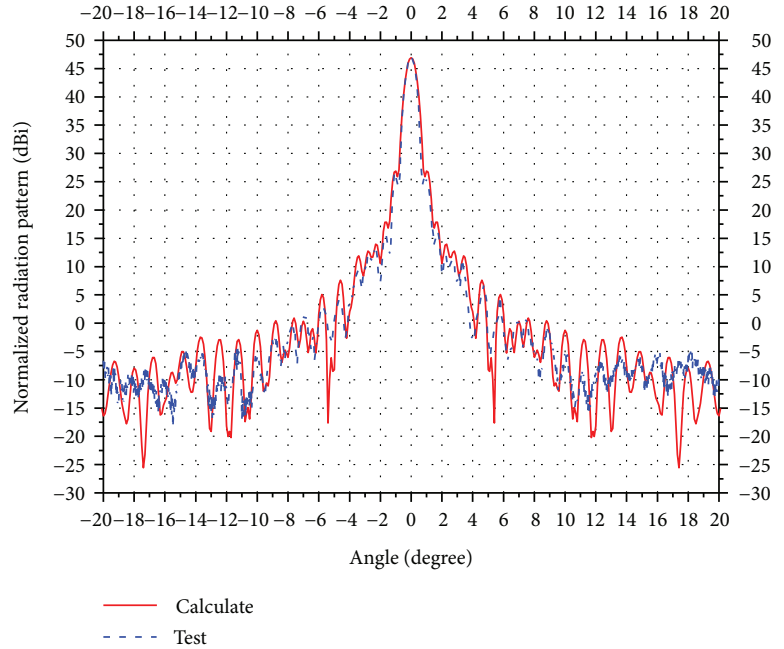

(a) $27.5 \mathrm{GHz}$

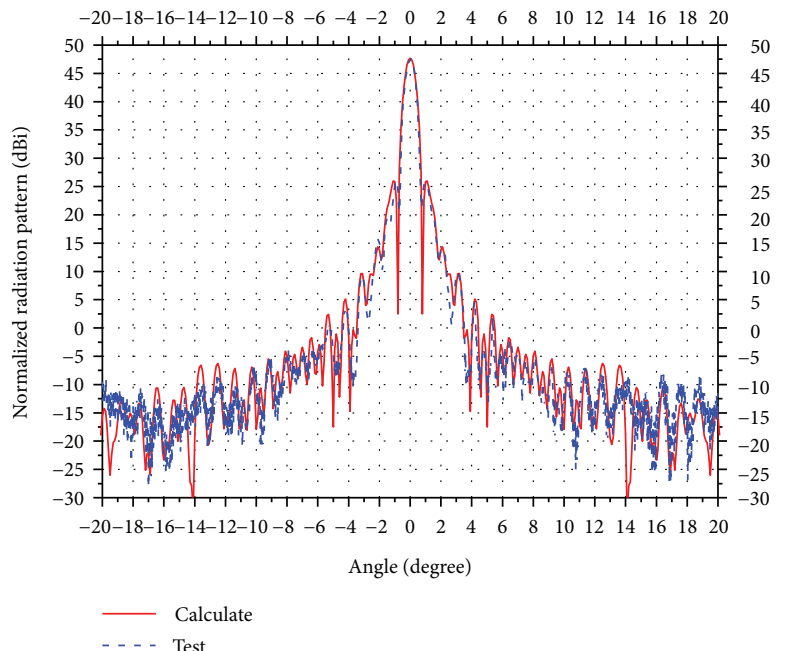

(c) $30.0 \mathrm{GHz}$

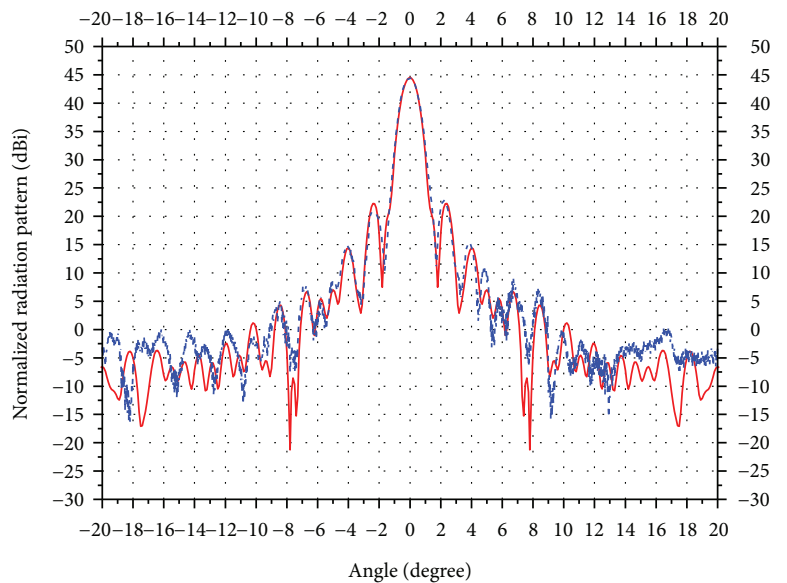

- Calculate
- . - Test

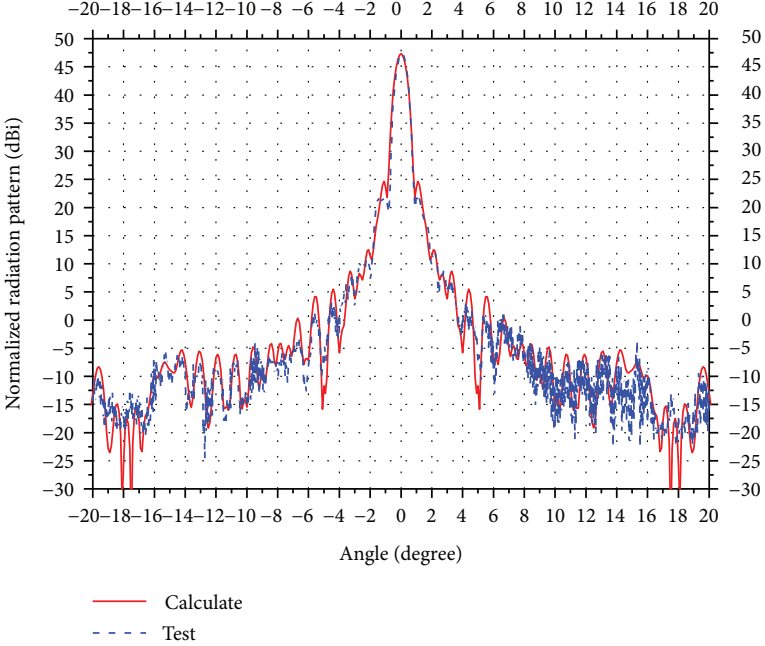

(b) $28.75 \mathrm{GHz}$

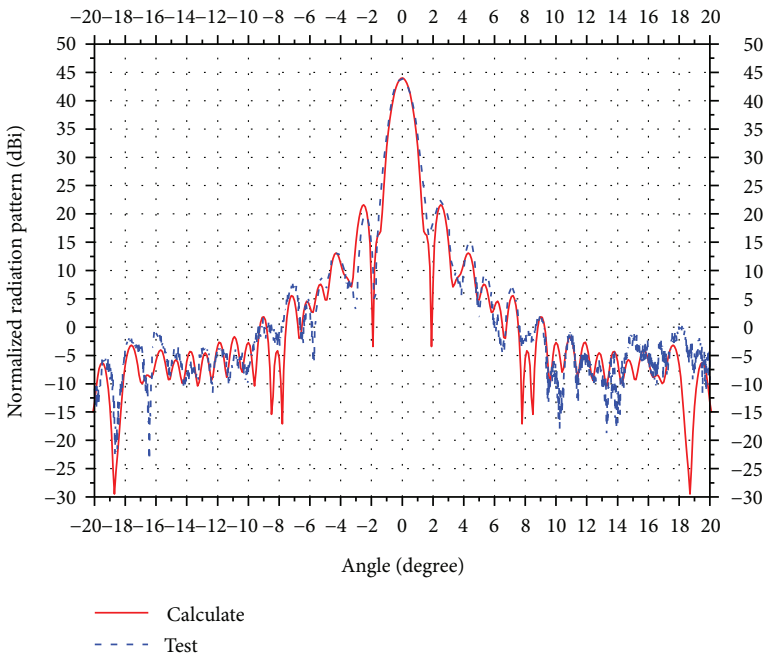

(d) $17.7 \mathrm{GHz}$

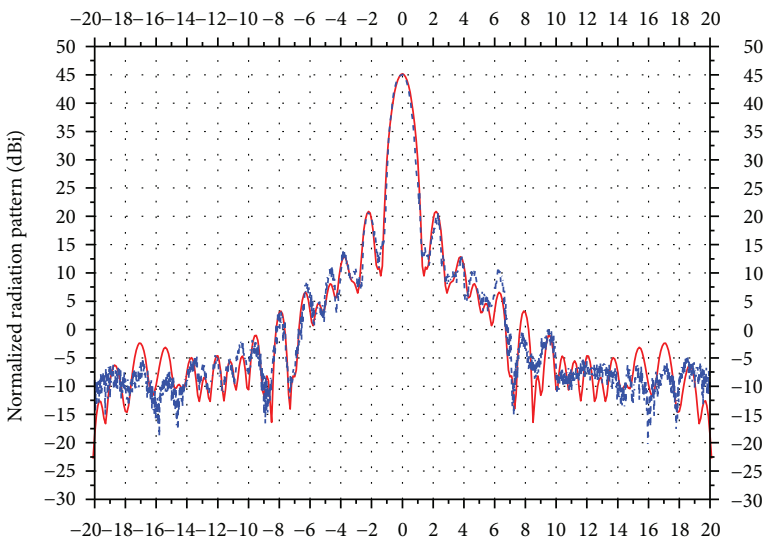

Angle (degree)

Calculate

(f) $20.2 \mathrm{GHz}$

(e) $18.95 \mathrm{GHz}$

Figure 8: Antenna pattern. 
TABLE 1: Measured data of antenna transmitting patterns.

\begin{tabular}{lccc}
\hline & $27.5 \mathrm{GHz}$ & $28.75 \mathrm{GHz}$ & $30 \mathrm{GHz}$ \\
\hline Gain $(\mathrm{dBi})$ & 47.57 & 47.92 & 48.63 \\
First sidelobe $(\mathrm{dB})$ & -20.04 & -22.65 & -21.66 \\
\hline
\end{tabular}

TABLE 2: Measured data of antenna receiving patterns.

\begin{tabular}{lccc}
\hline & $17.7 \mathrm{GHz}$ & $18.95 \mathrm{GHz}$ & $20.2 \mathrm{GHz}$ \\
\hline Gain $(\mathrm{dBi})$ & 43.79 & 44.27 & 44.89 \\
First sidelobe $(\mathrm{dB})$ & -22.4 & -22.28 & -24.31 \\
\hline
\end{tabular}

The VSWR is measured with Agilent Vector Network Analyzer N5230C in microwave anechoic chamber. After the measurement on antenna transmitting and receiving VSWR, the relative results are shown as blue chain lines in Figures 6 and 7.

As shown in Figure 6, antenna transmitting VSWR is below 1.24. As shown in Figure 7, antenna receiving VSWR is below 1.73 .

\section{Simulation and Measurement on Antenna Patterns}

This design adopts arbitrary phase and amplitude feed power pattern $P\left(\theta_{s}\right)$ as the feed model. Antenna patterns are simulated and analyzed by FEKO, and the curves obtained are shown with red continuous lines in Figure 8.

The far-field patterns are then measured, and the measured patterns obtained from data are shown as blue chain lines in Figure 8.

The distance between the transmitting and receiving antennas should satisfy the far-field test distance condition, namely,

$$
R \geq \frac{2 D^{2}}{\lambda},
$$

where $R$ is the distance between the source antenna and the antenna under test. $D$ is the aperture of the antenna under test, and $\lambda$ is the wavelength. According to (31), it is possible to calculate the minimum distance between the transmitting and receiving antennas in the far-field test. The highest frequency of the Ka-band antenna is $30 \mathrm{GHz}$, and $D=1020 \mathrm{~mm}$, so $R \geq 200 \mathrm{~m}$. The actual distance is about $282 \mathrm{~m}$.

The sidelobes meet the envelope of $29-25 \log \theta \mathrm{dBi}$. The measurement and related calculation of the antenna gain adopt comparison method. Acquired gain and first sidelobe are listed in Tables 1 and 2.

As shown in Figure 8, considering dielectric loss, splash plate scattering, cross-polarization efficiency, main reflector toleration, and other efficiency, simulated results of the antenna are highly consistent with the measured results, which demonstrates the success of such design. The measured antenna efficiency is about $65.6 \%$, while the efficiency of the existing antenna in Ka-band is below 60\% [16].

\section{Conclusion}

This paper introduces a ring-focus antenna based on splash plate feed. The prototype of this antenna has been produced based on the proposed design. The measured results verify that this antenna can achieve efficiency of over $65 \%$ and can satisfy the regulations of Rec. ITU-R S.580-6 in receiving band on antenna sidelobe. The measured results of the antenna are highly consistent with the simulated results, which proves the high engineering value of the proposed method.

\section{Conflicts of Interest}

The authors declare that there is no conflicts of interest regarding the publication of this paper.

\section{Acknowledgments}

This work was jointly sponsored by scientific research foundation NUPTSF (Grant nos. NY-214144 and NY-215073) and NSFC (Grant no. 61701260).

\section{References}

[1] T. Varum, J. N. Matos, V. Duarte, and P. Pinho, "Circularly polarized microstrip antenna array for the Ka-band," in 2015 IEEE International Symposium on Antennas and Propagation \& USNC/URSI National Radio Science Meeting, pp. 18641865, Vancouver, BC, Canada, 2015.

[2] A. Chen, Y. Zhang, Z. Chen, and C. Yang, "Development of a $\mathrm{Ka}$-band wideband circularly polarized 64-element microstrip antenna array with double application of the sequential rotation feeding technique," IEEE Antennas and Wireless Propagation Letters, vol. 10, pp. 1270-1273, 2011.

[3] A. Chen, Y. Zhang, Z. Chen, and S. Cao, "A Ka-band high-gain circularly polarized microstrip antenna array," IEEE Antennas and Wireless Propagation Letters, vol. 9, pp. 1115-1118, 2010.

[4] R. Glogowski, C. Peixeiro, J.-F. Zurcher, and J. R. Mosig, "Design and optimization of a shaped-beam ka-band substrate integrated waveguide antenna array," in 2015 Global Symposium on Millimeter Waves (GSMM), pp. 1-3, Montreal, QC, Canada, 2015.

[5] S. Chatterjee and A. Majumder, "Design of circularly polarized waveguide crossed slotted array antenna at Ka band," in 2015 International Conference on Microwave and Photonics (ICMAP), pp. 1-2, Dhanbad, India, 2015.

[6] A. Valero-Nogueira, J. I. Herranz-Herruzo, M. FerrandoRocher, R. Lenormand, A. Hirsch, and J.-L. Almeida, "Switchable RHCP/LHCP slotted waveguide array antenna for SATCOM on-the-move applications in Ka-band," in The 8th European Conference on Antennas and Propagation (EuCAP 2014), pp. 2047-2051, The Hague, Netherlands, April 2014.

[7] Y. Wang and A. M. Abbosh, "Software-defined reconfigurable antenna using slotted substrate integrated waveguide for Ka-band satellite-on-the-move communication," in 2015 International Symposium on Antennas and Propagation (ISAP), pp. 1-3, Hobart, TAS, Australia, 2015. 
[8] Y. Z. Liu, S. Xiao, and B. Z. Wang, "Analysis of a novel Ka horn antenna with low cross-polarization," Applied Computational Electromagnetics Society Journal, vol. 28, pp. 1061-1067, 2013.

[9] A. Rolland, N. T. Nguyen, R. Sauleau, C. Person, and L. Le Coq, "Smooth-walled light-weight Ka-band shaped horn antennas in metallized foam," IEEE Transactions on Antennas and Propagation, vol. 60, no. 3, pp. 1245-1251, 2012.

[10] D.-J. Lee, J.-Y. Kwon, N.-W. Kang, and J. F. Whitaker, "Reactive-to-radiating near-field propagation behavior measured on a Ka-band horn antenna," IEEE Antennas and Wireless Propagation Letters, vol. 10, pp. 1085-1087, 2011.

[11] Q. Lai, G. Almpanis, C. Fumeaux, H. Benedickter, and R. Vahldieck, "Comparison of the radiation efficiency for the dielectric resonator antenna and the microstrip antenna at Ka band," IEEE Transactions on Antennas and Propagation, vol. 56, no. 11, pp. 3589-3592, 2008.

[12] W. Fang, P. Fei, F. Nian, Y. Yang, and K. Feng, "Ka-band dielectric waveguide antenna array for millimeter wave active imaging system," Journal of Infrared, Millimeter, and Terahertz Waves, vol. 35, no. 11, pp. 962-973, 2014.

[13] Y. S. Kim, B. S. Kang, and B. J. Ku, "Design and measurements of Ka-band waveguide dielectric rod antenna for 7 channel digital beamformer for HAPS," in SympoTIC '04. Joint 1st Workshop on Mobile Future \& Symposium on Trends In Communications (IEEE Cat. No.04EX877), pp. 25-28, Bratislava, Slovakia, 2004.

[14] T. Gabriel, H. Kozilek, C. Hartwanger, M. Gerhard, and M. Schneider, "Steerable Ka-band dual reflector antenna," in 2016 German Microwave Conference (GeMiC), pp. 329-332, Bochum, Germany, March 2016.

[15] K. Kagoshima, S. Takeda, K. Ikeda et al., “A X/Ka bands feeder antenna for a planetary exploration high gain reflector antenna," in 2012 IEEE Antennas and Propagation Society International Symposium (APSURSI), pp. 1-2, Chicago, IL, USA, 2012.

[16] E. Curuk, K. Yegin, and C. Ozdemir, "A novel ultra-wide band design for feeding structure of Ka band VSAT parabolic reflector antenna," in 2016 21st International Conference on Microwave, Radar and Wireless Communications (MIKON), pp. 1-4, Krakow, Poland, 2016.

[17] R. Glogowski, J.-F. Zurcher, C. Peixeiro, and J. R. Mosig, "A low-loss planar Ka-band antenna subarray for space applications," IEEE Transactions on Antennas and Propagation, vol. 61, no. 9, pp. 4549-4557, 2013.

[18] Q. Luo, S. Gao, C. Zhang et al., "Design and analysis of a reflectarray using slot antenna elements for Ka-band SatCom," IEEE Transactions on Antennas and Propagation, vol. 63, no. 4, pp. 1365-1374, 2015.

[19] J. C. Guo, X. Q. Liu, W. Zhang, and B. Q. He, "Design of the Ka-band satellite antenna by parallel differential evolution," Applied Mechanics and Materials, vol. 556-562, pp. 21942197, 2014.

[20] S. Yun, M. Uhm, J. Choi, and I. Yom, "Multibeam reflector antenna fed by few elements for Ka-band communication satellite," in 2012 IEEE Antennas and Propagation Society International Symposium (APSURSI), pp. 1-2, Chicago, IL, USA, 2012.

[21] M. R. Chaharmir and J. Shaker, "Design of an FSS-backed 20/ $30 \mathrm{GHz}$ circularly polarized reflectarray for shared aperture X/ Ka-band satellite applications," in 2014 16th International Symposium on Antenna Technology and Applied Electromagnetics (Antem), p. 1, Victoria, BC, Canada, 2014.
[22] R. Wilke, S. Hamid, K. Schraml, R. Khunti, and D. Herberling, "Multi-layer patch antenna array design for Ka-band satellite communication," in 2013 SBMO/IEEE MTT-S International Microwave \& Optoelectronics Conference (IMOC), pp. 1-4, Rio de Janeiro, Brazil, 2013.

[23] A. Hoehn, P. B. Hager, and J. T. Harder, "Design characterization of an electronic steerable Ka-band antenna using liquid crystal phase shifters," in IEEE Aerospace Conference, pp. 1-14, Big Sky, MT, USA, March 2013.

[24] T. Smith, U. Gothelf, O. S. Kim, and O. Breinbjerg, "An FSSbacked $20 / 30 \mathrm{GHz}$ circularly polarized reflect array for a shared aperture L- and Ka-band satellite communication antenna," IEEE Transactions on Antennas and Propagation, vol. 62, no. 2, pp. 661-668, 2014.

[25] A. I. Sandhu, E. Arnieri, G. Amendola, L. Boccia, E. Meniconi, and V. Ziegler, "Radiating elements for shared aperture $\mathrm{Tx} / \mathrm{Rx}$ phased arrays at K/Ka band," IEEE Transactions on Antennas and Propagation, vol. 64, no. 6, pp. 2270-2282, 2016.

[26] H. Bayer, A. Krauss, T. Zaiczek, R. Stephan, O. EngeRosenblatt, and M. A. Hein, "Ka-band user terminal antennas for satellite communications [antenna applications corner]," IEEE Antennas and Propagation Magazine, vol. 58, no. 1, pp. 76-88, 2016.

[27] J.-M. Baracco, P. Ratajczak, P. Brachat, and G. Toso, "A dual frequency Ka-band printed Fresnel reflector for ground terminal applications," IEEE Transactions on Antennas and Propagation, vol. 63, no. 10, pp. 4352-4366, 2015.

[28] J. Yang and P.-S. Kildal, "Calculation of ring-shaped phase centers of feeds for ring-focus paraboloids," IEEE Transactions on Antennas and Propagation, vol. 48, no. 4, pp. 524-528, 2000.

[29] M. Van der Vorst and J. Gumpinger, "Applicability of 3D printing techniques for compact Ku-band medium/high-gain antennas," in 2016 10th European Conference on Antennas and Propagation (EuCAP), pp. 1-4, Davos, Switzerland, April 2016.

[30] T. Xiao-rong and S. Gao, "Design of low-cross-polarization wideband spaceborne ring focus reflector antenna," in 2014 Loughborough Antennas and Propagation Conference (LAPC), pp. 469-472, Loughborough, UK, November 2014.

[31] M. K. Kasraian, A. A. Kishk, and M. Tew, "Radiation characteristics of loaded shaped splash plate antennas," in Proceedings IEEE Southeastcon '92, vol. 1, pp. 306-309, Birmingham, AL, USA, April 1992.

[32] P. S. Kildal and A. Nyseth, "The hat feed: a new class of splashplate antenna having low cross-polarization," in 1986 Antennas and Propagation Society International Symposium, vol. 24, pp. 75-78, Philadelphia, PA, USA, June 1986.

[33] G. L. James and D. P. S. Malik, "Towards the theoretical design of splash-plate feeds," Electronics Letters, vol. 11, no. 24, pp. 593-594, 1975.

[34] C. Liu, S. Yang, and Z. Nie, "Design of a parabolic reflector antenna with a compact splash-plate feed," in 2013 Cross Strait Quad-Regional Radio Science and Wireless Technology Conference (CSQRWC), pp. 241-244, Chengdu, China, 2013.

[35] T. Inamura, "Upwash formation on splash plate atomization," International Journal of Multiphase Flow, vol. 85, pp. 67-75, 2016.

[36] Y.Asci, E. Curuk, K. Yegin, and C. Ozdemir, "Improved splashplate feed parabolic reflector antenna for Ka-Band VSAT applications," in 2016 46th European Microwave Conference (EuMC), pp. 1283-1286, London, UK, October 2016. 


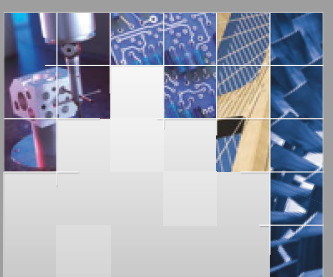

\section{Enfincering}
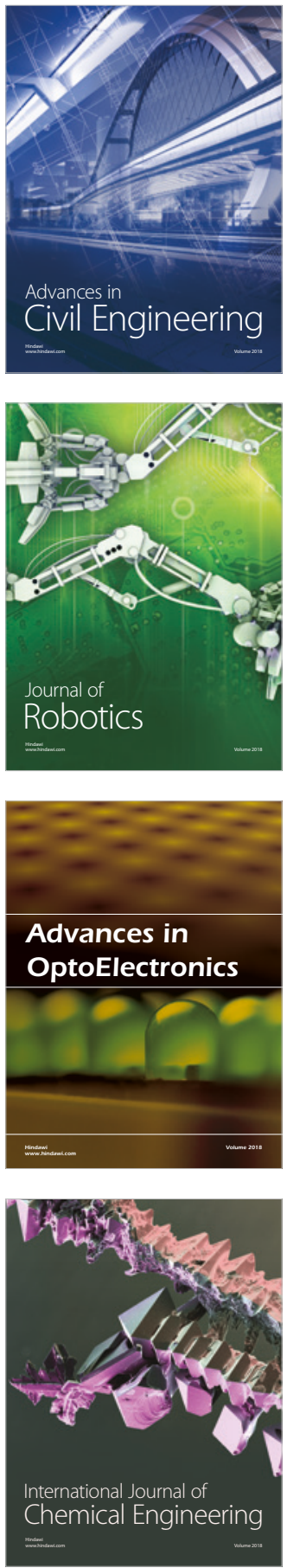

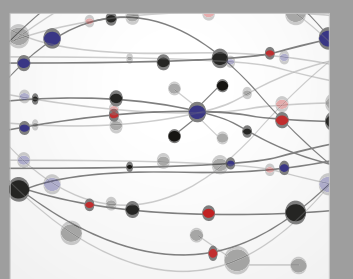

\section{Rotating \\ Machinery}

The Scientific World Journal

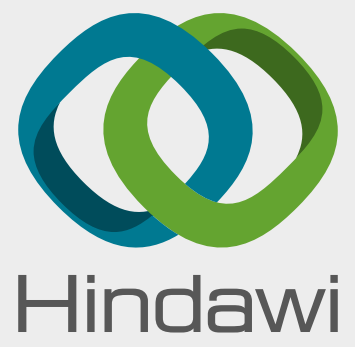

Submit your manuscripts at

www.hindawi.com
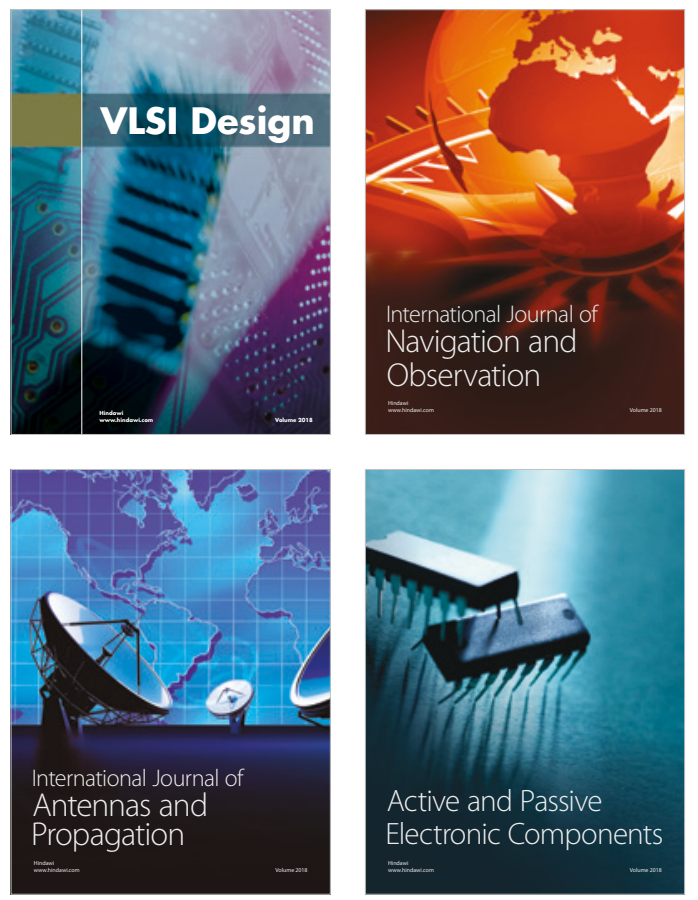
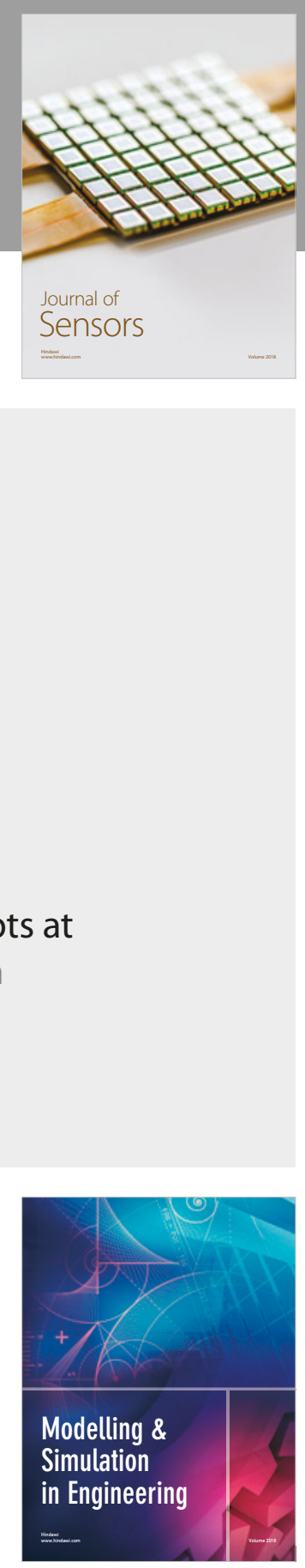

\section{Advances \\ Multimedia}
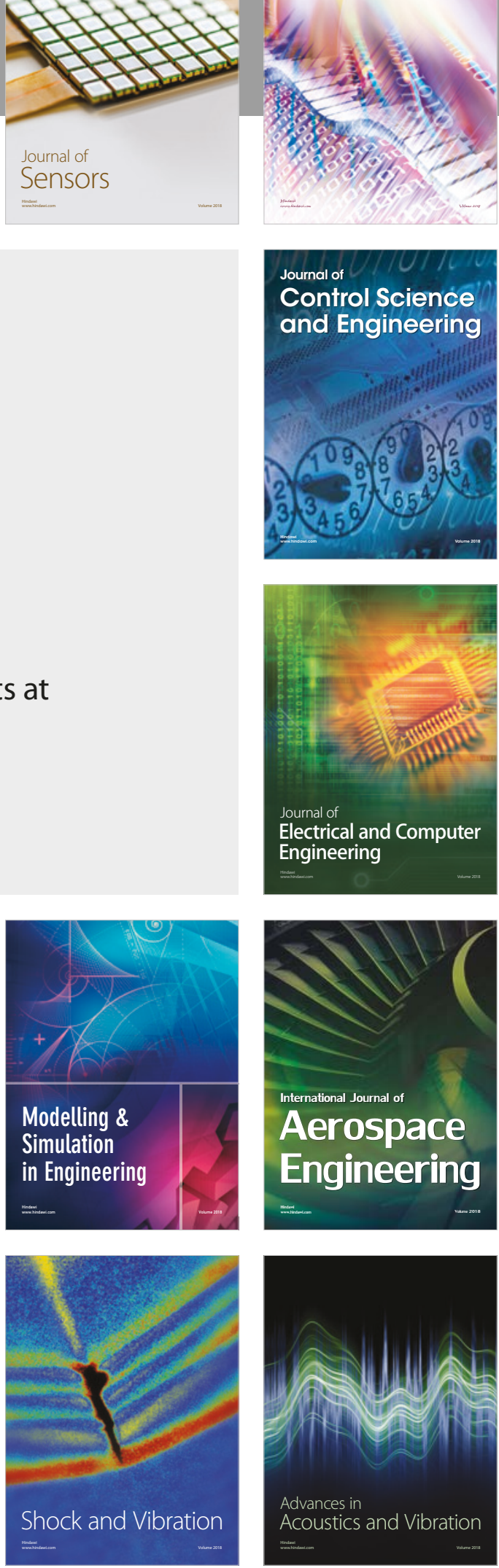